\title{
Review of: "Network centrality for the identification of biomarkers in respondent-driven sampling datasets"
}

Anupam Ghosh

Potential competing interests: The author(s) declared that no potential competing interests exist.

1. Methodology portion should be written much elaborately with a demonstration

2. Validation of the results needs to be incorporated

3. Overall its a good work 